\title{
Infrared standards for ISO
}

\section{A new calibration of mid infrared photometry}

\author{
P.L. Hammersley ${ }^{1}$, M. Jourdain de Muizon ${ }^{2,3,7, \star}$, M.F. Kessler ${ }^{4, *}$, P. Bouchet ${ }^{5}$, R.D. Joseph ${ }^{6}$, \\ H.J. Habing ${ }^{7}$, A. Salama ${ }^{4, *}$, and L. Metcalfe ${ }^{4}$ \\ 1 Instituto de Astrofísica de Canarias, E-38200 La Laguna, Tenerife, Spain \\ 2 DESPA, Observatoire de Paris, Meudon, France \\ ${ }^{3}$ LAEFF, VILSPA, P.O. Box 50727, 28080 Madrid, Spain \\ 4 ESA, VILSPA, P.O. Box 50727, 28080 Madrid, Spain \\ ${ }^{5}$ European Southern Observatory, Casilla 19001, Santiago 19, Chile \\ ${ }^{6}$ IRTF, Institute for Astronomy, 2680 Woodlawn Drive, Honolulu, HI96822, U.S.A. \\ 7 Leiden Observatory, Leiden University, P.O. Box 9513, 2300 RA Leiden, The Netherlands
}

Received January 13; accepted June 17, 1997

\begin{abstract}
We present $N, 8.7 \mu \mathrm{m}, 9.8 \mu \mathrm{m} 12.5 \mu \mathrm{m}$ and $Q$ bolometry of 26 stars which were observed on the IRTF. These are primarily $\mathrm{K}$ giants which have been well measured at other wavelengths and are chosen to be single and non-variable. Hence the data presented provides a new homogeneous set of mid IR standards. As a test of part of the calibration strategy of ISO over this wavelength range, the zero points have been set using the Kurucz model grids (1993) to predict the mid IR magnitudes from near IR data. An analysis of the errors involved is presented and a comparison with more direct methods for the determination of the zero points suggest an possible error of 0.015 mags, although the source of this error is unclear as it is at the level of the uncertainty in the more direct methods. The effect of the $\mathrm{SiO}$ fundamental on the spectral energy distributions of $\mathrm{K}$ giants is discussed.
\end{abstract}

Key words: techniques: photometric - stars: fundamental parameters - infrared: stars

\section{Introduction}

The 10 and $20 \mu \mathrm{m}$ observations and the results presented in this paper were performed and obtained as part of the Infrared Space Observatory's (ISO) calibration programme.

Send offprint requests to: P.L. Hammersley

* Visiting Astronomer at the Infrared Telescope Facility which is operated by the University of Hawaii under contract to the National Aeronautics and Space Administration.
The ISO ground-based preparatory programme working group (ISO-GBPPwg), chaired by H.J. Habing, set up the goal in 1990 to characterise accurately in the infrared some 400 stars covering the whole sky, as well as a wide range of spectral type and brightness (Jourdain de Muizon \& Habing 1991). We have thus obtained $J, H, K$, and $L$ narrow-band photometry for all stars and for the brightest ones, spectroscopy and/or $N$ and $Q$ bolometry (van der Bliek et al. 1996; Hammersley et al., this work and in preparation).

The stellar effective temperatures $\left(T_{\mathrm{e}}\right)$ were determined using on one hand the Infrared Flux Method (IRFM, Blackwell et al. 1990, 1995), and on the other hand the $V-K$ versus $T_{\text {e }}$ relationship (Di Benedetto 1993, 1995). Both methods were combined with the surface gravity and metallicity (Cayrel de Strobel et al. 1992) to predict stellar spectra up to about $50 \mu \mathrm{m}$ from the Kurucz stellar model atmosphere grids (Kurucz 1993).

The 10 and $20 \mu \mathrm{m}$ bolometry was originally planned to be used to test the model atmospheres and detect stars with infrared excesses (hence would be unsuitable as ISO IR standards). However, it turned out that, due to the lack of reliable mid-infrared standards, the 10 and $20 \mu \mathrm{m}$ observations could not be accurately flux calibrated using the standard methods and so serve the original purpose.

In Sect. 2 we describe the star selection and the observations. In Sect. 3 we present the data reduction, in particular tackling the delicate problem of determining a zero-point. We describe the use of the models and evaluate the contribution of all the possible sources of errors. In Sect. 4, we discuss the validity of using models to extrapolate mid-infrared magnitudes from near-infrared data, thus producing a new set of mid-infrared standards. 


\section{Source selection and observations}

The star list was compiled from a larger list of stars for which accurate near infrared narrow-band photometry had previously been obtained (Selby et al. 1988; Hammersley et al. 1997). The stars were chosen as single and non variable, with spectral type in the range K9 to A0 and luminosity class III to V. However, in practice, the stars observed for this paper are $\mathrm{K}$ and $\mathrm{G}$ giants as these are the only suitable sources which are sufficiently bright to give a high signal to noise ratio at $10 \mu \mathrm{m}$. Their $V$ magnitudes are typically between 3 and 5.5 and their $K$ magnitudes between -3 and 2 . A list of fainter stars ( $K$ between 2 and 3 ) was also planned but could not be observed, with one exception, due to lack of time. All the data necessary to apply the IRFM on these stars were then already available.

The observations were performed at the Infrared Telescope Facility (IRTF) on Mauna Kea (Hawaii) during four nights between February 25th and March 1st, 1992. The instrument used was the facility helium cooled bolometer.

The weather conditions were excellent during the four nights with a seeing of 1 arcsec or better, and relative atmospheric humidity of $1 \%$. Programme stars were always observed at airmass between 1.0 and 1.3. The stars were measured in five filters, namely the two broad-band $N$ and $Q(10$ and $20 \mu \mathrm{m})$ and three narrow-band filters $8.7 \mu \mathrm{m}$ $9.8 \mu \mathrm{m}$ and $12.5 \mu \mathrm{m}$. A beam size of 5 arcsec and a chopper throw of 15 arcsec were used. The integration time was 10 seconds and 6,12 or 24 cycles were performed in each filter.

A set of 24 programme stars could be observed during the February 92 run. Nine of them were measured twice, on two different nights. Six IRTF standards were observed several times during the run: HR 1457 ( $\alpha$ Tau), HR 2061 ( $\alpha$ Ori), HR 2990 ( $\beta$ Gem), HR 4069 ( $\mu$ UMa), HR 4534 ( $\beta$ Leo) and HR 5340 ( $\alpha$ Boo). These were used to calibrate the atmospheric transmission and instrumental response but not the final flux calibration as will be discussed later. Two of these stars are programme stars bringing the number of stars in the present sample to 26 . The main parameters of these stars are listed in Table 1. Unfortunately, the stars in this paper cover only the range of R.A. from 6 to 18 hours. A second run was planned and allocated for early September 1992, almost exactly six months apart from the first run, in order to complete the sky coverage. Unfortunately, the weather conditions were so bad then that not a single additional star could be observed.

\section{Data reduction and analysis}

\subsection{Deriving the apparent magnitudes}

Originally it was hoped to reduce the data using well calibrated standard stars, as would be the case with near IR data sets. However, it quickly became apparent that in general there is a lack of 10 and $20 \mu \mathrm{m}$ data with known pass bands and a known zero points, particularly for the narrow band filters. For a summary of the current situation in 10 and $20 \mu \mathrm{m}$ photometry see Hanner \& Tokunaga (1991). It was therefore decided to set up new zero points for the IRTF filter set. The zero point that was adopted was to define that the photospheric Vega (HR 7001) is 0.00 magnitudes in all filters. The magnitudes of the stars measured were then tied to this zero point using the Kurucz 1993 model atmosphere grid.

The method used compares the predicted in-band flux density for each star in each filter with the measured signal. The in-band flux density $(E)$ is

$E=\int_{0}^{\infty} S_{\lambda} f_{\lambda} \mathrm{d} \lambda$

where $f_{\lambda}$ is the spectral energy distribution (SED) of the star $S_{\lambda}$ is the total transmission function for the entire system, i.e. the multiplication of the filter profile, detector response and the terrestrial atmospheric transmission function.

\subsubsection{Initial flux densities}

The programme RED (developed at ESO Chile by P. Bouchet) was used to remove the differential atmospheric extinction to provide a table whose values are proportional to the flux density of the star in each filter. The programme also calculates an error for each measurement based on the fluctuations in the measured signals and the accuracy of the airmass calibration.

\subsubsection{The total system transmission}

The total system transmission is the multiplication of the detector response, the filter profiles and the atmospheric transmission above Mauna Kea. The detector response was ignored as the response of a bolometer is fairly flat over the wavelength range of interest. The filter profiles come from cold scans of an identical set of filters provided by OCLI to UKIRT, the IRTF and the University of Minnesota (Cohen 1995b), whilst the atmospheric transmission is that used in Cohen et al. (1992). Figure 1 shows the adopted transmission function for the 5 filters.

\subsubsection{The absolute photospheric Vega model}

Tokunaga (1984) shows that there is no evidence of an IR excess in Vega in the $N-Q$ colour. However, part of the aim of this project is to assess the possibility of predicting IR flux densities to beyond $30 \mu \mathrm{m}$ using the model SEDs where the IR excess of Vega (Aumann et al. 1985) would be significant. Hence the true Vega flux density cannot be used as the zero point for the longest wavelengths. We have therefore chosen to use a photospheric Vega model as the zero point. 
Table 1. The main parameters of the stars observed

\begin{tabular}{|c|c|c|c|c|c|c|c|c|}
\hline name & $\mathrm{RA}(2000)$ & $\overline{\text { Dec }}$ & $\begin{array}{l}\text { Spectral } \\
\text { type }\end{array}$ & $T_{\mathrm{e}}$ & $m_{V}$ & $m_{K}$ & $\begin{array}{r}\text { IRAS } 12 \mu \mathrm{m} \\
(\mathrm{Jy}) \\
\end{array}$ & $\begin{array}{r}\text { IRAS } 25 \mu \mathrm{m} \\
(\mathrm{Jy})\end{array}$ \\
\hline$\overline{\mathrm{HR}} 1457$ & $04: 35: 55.2$ & $+16: 30: 33$ & K5III & 3920 & 0.87 & -2.85 & 699.70 & 153.30 \\
\hline HR 2077 & $05: 59: 31.6$ & $+54: 17: 05$ & K0III & 4795 & 3.72 & 1.43 & 11.68 & 2.68 \\
\hline HR 2335 & $06: 27: 15.5$ & $-00: 16: 34$ & K5III & 4180 & 5.54 & 2.42 & 5.01 & 1.17 \\
\hline HR 2443 & $06: 37: 53.3$ & $-18: 14: 15$ & KIII1 & 4589 & 4.42 & 1.75 & 8.61 & 2.15 \\
\hline HR 2459 & $06: 43: 04.9$ & $+44: 31: 28$ & K5III & 4035 & 5.02 & 1.54 & 11.14 & 2.71 \\
\hline HR 2533 & $06: 51: 59.9$ & $+23: 36: 06$ & K5III & 4084 & 5.67 & 2.30 & 5.83 & 1.42 \\
\hline HR 2970 & $07: 41: 14.8$ & $-09: 33: 04$ & KOIII & 4813 & 3.94 & 1.61 & 10.23 & 2.37 \\
\hline HR 3145 & $08: 02: 15.9$ & $+02: 20: 04$ & K2III & 4289 & 4.39 & 1.42 & 12.22 & 2.91 \\
\hline HR 3304 & $08: 26: 27.6$ & $+27: 53: 36$ & K5III & 4162 & 5.57 & 2.35 & 5.22 & 1.31 \\
\hline HR 3738 & $09: 25: 24.0$ & $-05: 07: 03$ & K5III & 3972 & 5.59 & 2.06 & 7.41 & 1.74 \\
\hline HR 3834 & $09: 38: 27.2$ & $+04: 38: 57$ & K3III & 4180 & 4.68 & 1.52 & 10.88 & 2.63 \\
\hline HR 3939 & 09:59:51.6 & $+56: 48: 42$ & K5III & 4052 & 5.49 & 2.04 & 6.91 & 1.76 \\
\hline HR 4094 & $10: 26: 05.4$ & $-16: 50: 11$ & K4.5III & 4054 & 3.82 & 0.34 & 31.59 & 7.56 \\
\hline HR 4232 & $10: 49: 37.4$ & $-16: 11: 37$ & K2III & 4349 & 3.11 & 0.25 & 35.08 & 8.25 \\
\hline HR 4335 & 11:09:39.7 & $+44: 29: 54$ & KIII1 & 4597 & 3.00 & 0.41 & 31.02 & 7.41 \\
\hline HR 4402 & $11: 24: 36.5$ & $-10: 51: 34$ & K5III & 3859 & 4.82 & 0.95 & 18.29 & 4.46 \\
\hline HR 4701 & $12: 20: 50.7$ & $+57: 51: 50$ & K5III & 4060 & 5.53 & 2.09 & 6.57 & 1.68 \\
\hline HR 4728 & $12: 25: 50.9$ & $+39: 01: 07$ & G9III & 4987 & 5.02 & 2.94 & 3.11 & 0.81 \\
\hline HR 4954 & $13: 07: 10.6$ & $+27: 37: 29$ & K5III & 4022 & 4.81 & 1.32 & 14.25 & 3.45 \\
\hline HR 5315 & $14: 12: 53.7$ & $-10: 16: 25$ & K3III & 4232 & 4.18 & 1.00 & 17.90 & 4.25 \\
\hline HR 5340 & $14: 15: 39.6$ & $+19: 10: 57$ & K1III & 4321 & -0.05 & -3.05 & 793.10 & 163.05 \\
\hline HR 5616 & $15: 04: 26.7$ & $+26: 56: 51$ & K2III & 4356 & 4.54 & 1.64 & 10.68 & 2.53 \\
\hline HR 5622 & $15: 06: 37.5$ & $-16: 15: 24$ & K5III & 3896 & 5.18 & 1.45 & 12.05 & 2.90 \\
\hline HR 6108 & $16: 22: 29.1$ & $+33: 42: 13$ & K5III & 3948 & 5.40 & 1.71 & 9.51 & 2.32 \\
\hline HR 6159 & $16: 32: 36.2$ & $+11: 29: 17$ & K7III & 3952 & 4.84 & 1.22 & 15.02 & 3.71 \\
\hline HR 6705 & $17: 56: 36.3$ & $+51: 29: 20$ & K5III & 3986 & 2.23 & -1.31 & 155.10 & 37.84 \\
\hline
\end{tabular}

There remains some controversy over what is the absolute Vega flux density in the infrared, a discussion of which is provided in Mégessier (1995) or Cohen et al. (1992). However, the black body approximation to Vega suggested by Mégessier is unacceptable in this case as it is not clear that the approximation is applicable at $25 \mu \mathrm{m}$. Further the zero points are to be set up using the Kurucz model grids to tie the measured stars to Vega; therefore it is preferable to use a Vega model also derived from the same source.

The photospheric Vega model that we have chosen to adopt is that presented in Cohen (1992), which is a customised Kurucz model that incorporates the metal poor character of Vega. This has a nominal error of $1.45 \%$ and in general is within a few percent of other determinations in the near IR. Over the IRTF filter range the flux densities agree with the values given in Hanner \& Tokunaga (1991) to within the errors. For the purpose of this paper we shall ignore the error in the Vega flux density and take the model as being a definition. When better determinations become available it is simple to update the given flux densities.
3.1.4. The spectral energy distributions for the stars and predicted magnitudes

A spectral energy distribution was derived for each star measured in this observing programme. It was decided to base the SEDs on the Kurucz 1993 model grids because they cover a wide range of effective temperatures $\left(T_{\mathrm{e}}\right)$, metallicities and surface gravities and have a sufficiently high spectral resolution in the near IR that the majority of the major stellar features (e.g. CO) are clearly visible. This grid is derived using the ATLAS 9 code Kurucz (1991).

The stars already have accurate near IR and visible magnitudes Selby et al. (1988); Hammersley et al. (1997), Van de Bliek et al. (1996). The effective temperatures are based on Blackwell et al. (1991). For the stars in common with Blackwell et al. (1991) the $T_{\mathrm{e}}$ was used directly, whilst for the remainder the $T_{\mathrm{e}}$ was derived from the $V-K$ versus $T_{\mathrm{e}}$ relationship, such as that presented in Fig. 4 of Blackwell et al. (1990). The accuracy of such a determination of $T_{\mathrm{e}}$ is comparable to that of the IRFM and the dominant errors will be systematic. These effective temperatures were then used with the surface gravity and metallicity (Cayrel de Strobel et al. 1992) to extract a spectral shape from the Kurucz model grid. When a log $g$ was not available it was assumed from the spectral type 

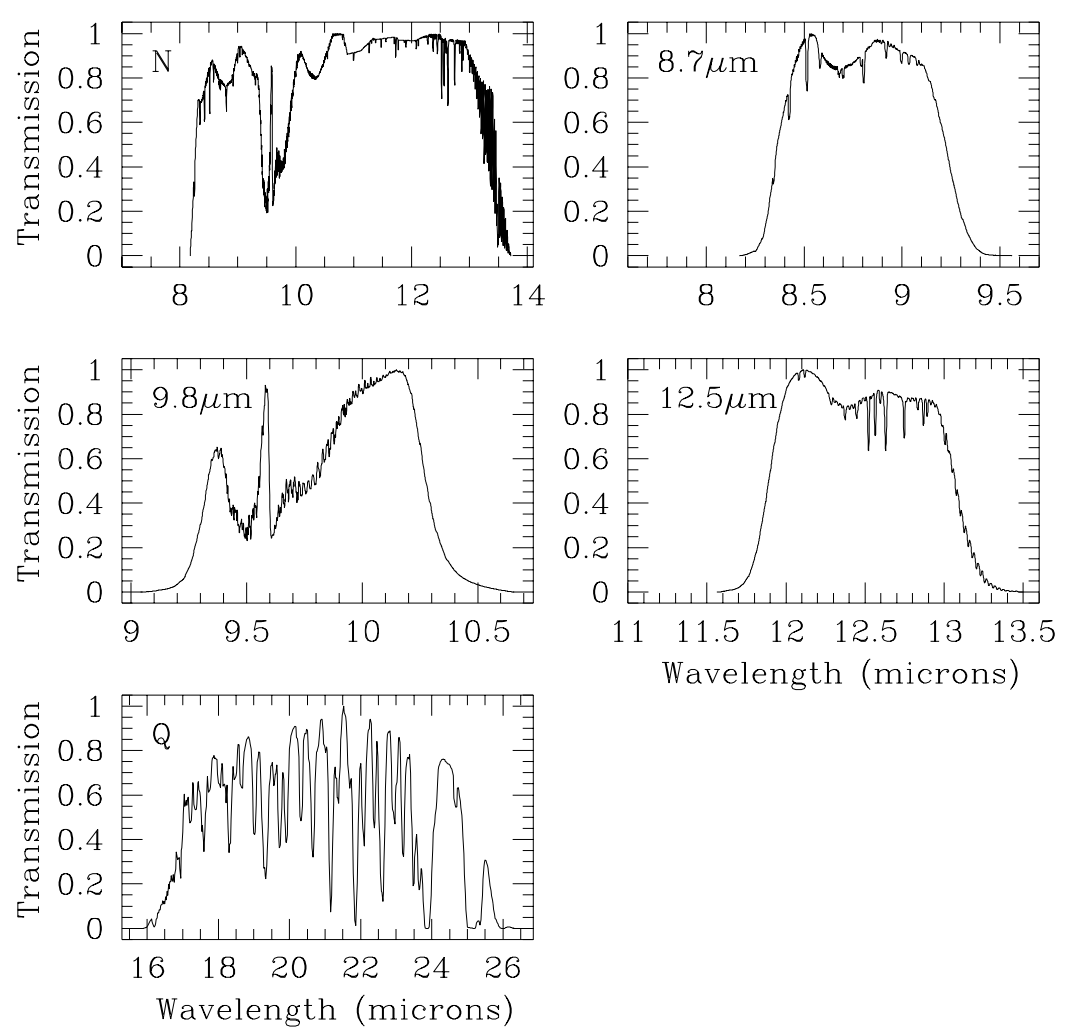

Fig. 1. The total transmission curves for the 5 filters

and when a metallicity was not available it was assumed to be solar.

The SEDs needed to calibrate the IRTF bolometry are only required for $\mathrm{G}$ to $\mathrm{K}$ giants between about 8 and $25 \mu \mathrm{m}$. Even for the more general case of calibrating ISO the required SEDs are for A0 to M0 dwarfs and giants at wavelengths longer than $2.5 \mu \mathrm{m}$. In both cases the stars are close to the Rayleigh-Jeans part of their flux curves in the wavelength range of interest and the opacities are dominated by $\mathrm{H}^{-}$free-free. Therefore, extracting the SEDs was done in such a way that the error in the wavelength range of interest would be minimised.

The final error in a SED is made up of a number of factors.

Error in the initial photometry. The spectral shapes are turned into absolute flux densities by normalising to filter photometry. This photometry will have an error, typically of order $1 \%$, or better, for high quality $V R I J H K$ data but 2 or $3 \%$ at $L$ and $M$.

The error in $\boldsymbol{T}_{\mathrm{e}}$. There is an error in the derived $T_{\mathrm{e}}$ of 1.5 to $3 \%$ (Blackwell et al. 1994) which will lead to a wavelength dependent error in the shape of the SED. The dominant source of this error is a systematic difference in derived $T_{\mathrm{e}}$ depending on which model grid is adopted (Mégessier 1994)
In effect the method used here predicts a colour from the shape of the SED. Hence, the magnitude in one filter is obtained using the measured magnitude in the other filter and the predicted colour. However, as the shape of the SED has a temperature dependent error, the predicted colour will also have an error which will depend on the $T_{\mathrm{e}}$ and the filters chosen. Figure 2 shows the difference in 5 colours when $T_{\mathrm{e}}$ changes by $2 \%$ at $4100 \mathrm{~K}, 5000 \mathrm{~K}, 6000 \mathrm{~K}$ and $9000 \mathrm{~K}$. The longest wavelength is in all cases $L$, so that real measurements could be used. At these effective temperatures, $L$ is nearly on the Rayleigh-Jeans part of the stellar spectra. Therefore the $L-N$ colour will be within a few percent of zero and the values in Fig. 2 would be almost identical if a longer wavelength than $L$ were used. The JHKL data were taken from Van der Bliek et al. (1996) and the $B V$ data from the Hipparcos Input Catalogue (Turon et al. 1992). Curves for each colour against $T_{\mathrm{e}}$ were calculated using a polynomial fit. The colour at each $T_{\mathrm{e}}$, and then $2 \%$ different, were then determined from the fits. As can be seen when both filters are close to the Rayleigh-Jeans part of the spectrum $(K-L$ for all four temperatures and $J H K-L$ at $9000 \mathrm{~K})$ the colour is insensitive to small changes in temperature. However, if one, or indeed both, filters are far from the Rayleigh-Jeans part of the spectrum 
then the change in $T_{\mathrm{e}}$ will produces a significant change in the derived colour. Hence, an error in $T_{\mathrm{e}}$ of $2 \%$ would give an error in predicted $J-L$ of around $4-5 \%$ for the stars presented here.

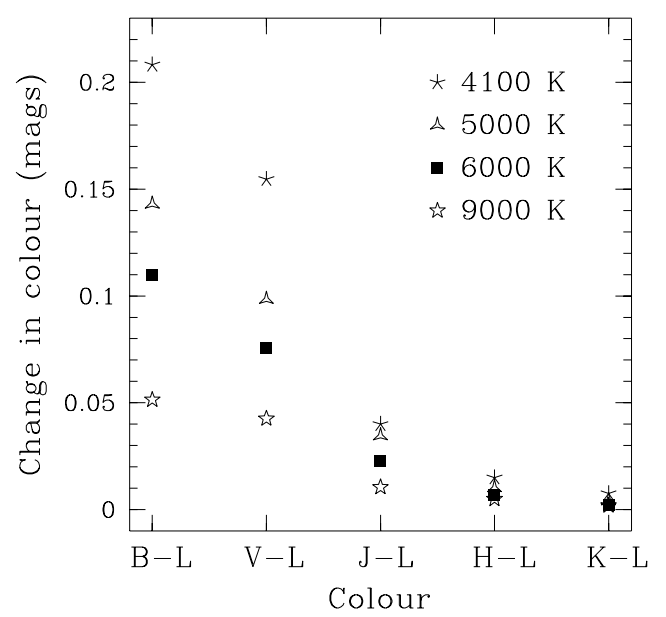

Fig. 2. The change in colour when the $T_{\mathrm{e}}$ is changes by $2 \%$

In order to minimise the error in the SED longwards of $2.5 \mu \mathrm{m}$, the filters used to normalise the spectra must lie close to the Rayleigh-Jeans part of the star's spectrum. For the data set presented here this means that only filters with a wavelength longer than $H$ should be used. If $K$ is taken as the basis filter, then an error of $3 \%$ in $T_{\mathrm{e}}$ at $4100 \mathrm{~K}$ (typical for the used stars here) would produce an error of 0.004 mags in the predicted $Q$ magnitude.

The error in metallicity and Log $\boldsymbol{g}$. Close to the Rayleigh-Jeans part of the spectra the shape of the SEDs are, in general, insensitive to small changes in metallicity. The stars used will be almost exclusively disc stars, as opposed to low metallicity halo stars, so for those stars without a metallicity the error will be of the order \pm 0.3 dex which will cause a negligible error in the SED. In the same manner the SEDs are insensitive to small changes in $\log g$.

Errors in the models. The models can only be considered to be approximations to the true SED of the stars, for example note the controversy over the Vega flux density. However, there are specific regions in the spectrum which could be particularly difficult to model. One example could be in the $H$ window where the spectra change from being dominated by the $\mathrm{H}^{-}$boundfree opacity to the $\mathrm{H}^{-}$free-free opacity. This causes a knee in the SED of cooler stars at $1.6 \mu \mathrm{m}$. A full discussion of this is beyond the scope of the paper. However, the error in the predicted colour is likely to be less if two wavelengths are dominated by the same opacity. As we are interested in wavelengths between 8 and $25 \mu \mathrm{m}$ then the wavelengths used to normalise the SED should be longer then $1.6 \mu \mathrm{m}$ implying that $K$ should be the shortest filter to be used.

From the above discussion it is clear that if a weighted mean, using the near IR filters, is used to normalise the SED of K giants, then the $K$ filter will be by far the most important. The $J$ filter would have too large an error because of the uncertainty in the $T_{\mathrm{e}}$ and the $L$ filter has a significantly larger error in the photometry. In order to simplify matters it was decided to use only the $K$ filter to normalise the SEDs. As up to 26 stars are used to set the zero point, using just the $K$ filter will not significantly influence the final error.

Once the SED has been normalised, the predicted magnitude for a star in each of the IRTF filters is then

$p m_{s, i}=-2.5\left(\log E_{s, i}-\log E_{\mathrm{vega}, i}\right)$

where $p m_{s, i}$ is the predicted magnitude of star $s$ in filter $i$.

\subsection{Calculation of the system constant}

In principle the system constant $(S C)$ for each filter is then

$S C=\frac{\sum p m_{s}+2.5 \log \left(V_{\mathrm{s}}\right)}{n}$

where $p m_{s}$ is the predicted flux density for star $s$.

$V_{\mathrm{s}}$ is the measured signal after being corrected for the atmospheric extinction. $n$ is the number of stars.

The main calibration stars are bright, well measured at all wavelengths and were measured a number of times. Therefore, it was decided to work with the individual measurements to weight the result in favour of the most measured stars.

Figure 3 shows the system constant (i.e. $p m_{s}+$ $\left.2.5 \log \left(V_{\mathrm{s}}\right)\right)$ against temperature for every measurement in the 5 filters. In general the scatter is small, however there are a few problems.

\subsubsection{The $\mathrm{SiO}$ bands}

The Kurucz model grids, which were used for constructing the SEDs, do not contain the $\mathrm{SiO}$ absorption bands. These affect the region between about 7.8 and $11 \mu \mathrm{m}$ in cool stars, such as many of those observed in this data set. The result is that the system constant calculated for these stars will be too low over this wavelength range. The effect of the $\mathrm{SiO}$ can be clearly seen in the $N, 8.7 \mu \mathrm{m}$ and, to a lesser extent, the $9.8 \mu \mathrm{m}$ filters for temperatures cooler than about $4400 \mathrm{~K}$. From spectroscopy is clear that there is still a small amount of $\mathrm{SiO}$ in stars as hot as $4900 \mathrm{~K}$, however, Fig. 1 suggests that the $\mathrm{SiO}$ apparently disappears in these filters for stars hotter than about $4500 \mathrm{~K}$. Hence in order to determine the system constant in the $N, 8.7 \mu \mathrm{m}$ and $9.8 \mu \mathrm{m}$ filters only those stars hotter than 


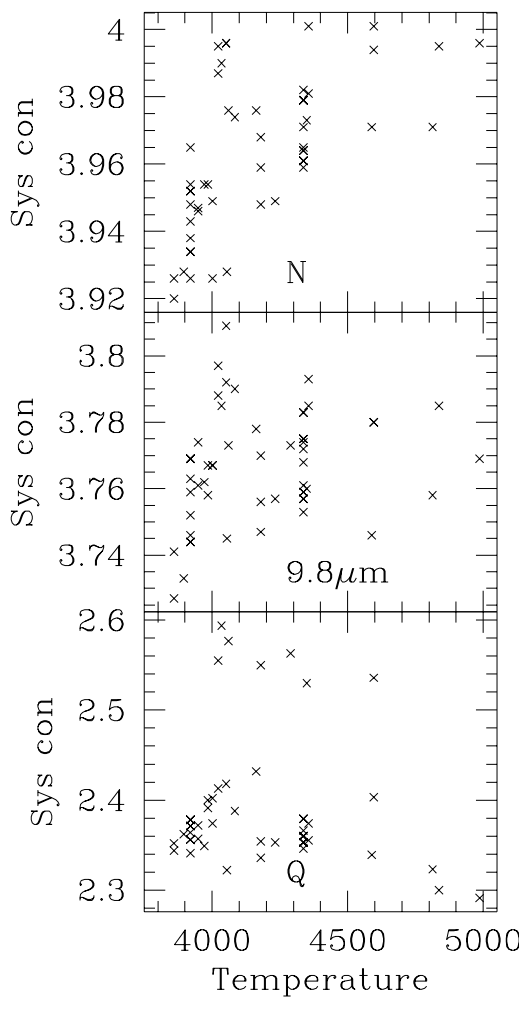

Fig. 3. The system constants in the 5 filters

$4500 \mathrm{~K}$ have been used. The residual $\mathrm{SiO}$ could lead to a small error, but probably well under 0.01 magnitudes. The effect of the $\mathrm{SiO}$ is not significant in the other filters so all of the measurements have been used.

\subsubsection{Bad points}

There are a few obvious bad points which were deleted. However, there are a couple of anomalies.

In the $Q$ filter there are a group of points with a system constant near 2.55, well away from the rest of the group. It turns out these are all of the points that were taken on the 26th Feb., except one. The exception was a HR 5340 point which was used to calibrate the day. It was decided that HR 5340 measurement was in error, so it was deleted, and the remaining $Q$ values on that day had 0.195 mags subtracted.

In the $12.5 \mu \mathrm{m}$ filter there are 7 points which are near 3.07. Two of these stars were re-measured and the values were the expected ones. The other five were only measured once. The points come from different days and there is no clear indication of a cause. As none of these points belong to HR 5340 or HR 1457 which were the main calibration sources, and as the magnitudes for these stars in the other filters are as expected, these points have been deleted from the $12.5 \mu \mathrm{m}$ filter set.

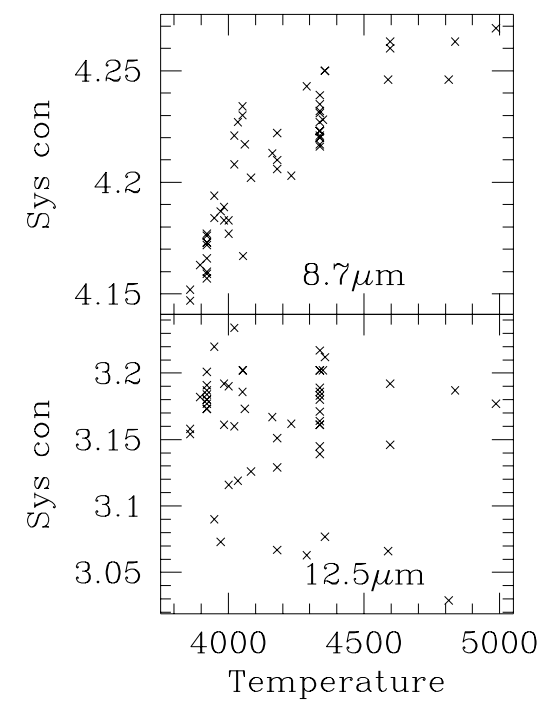

\subsubsection{Calculated system constants}

The after taking acount of the above points, the calculated system constants are

$N=3.990 \pm 0.007$
$8.7=4.260 \pm 0.007$
$9.8=3.772 \pm 0.007$

$12.5=3.185 \pm 0.004$

$Q=2.362 \pm 0.006$.

The quoted error is the standard error of the distribution.

\subsection{The final magnitudes}

The magnitude $(m)$ for each observation is calculated in the normal way for each filter, i.e.:

$m_{s}=S C-2.5 \log \left(V_{\mathrm{s}}\right)$

The final magnitudes are presented in Table 2. When there are multiple observations of a star these have been averaged using an inverse variance weighted mean and the error quoted is the standard error from the individual values. When there is only one measurement the error is calculated from the error in the original measurement or 0.01 which ever is the greater. The error given at this stage does 
Table 2. The magnitudes in the 5 IRTF filters. Given are the magnitude, error and number of measurements. Note the error does not include the error in the zero points and in general 0.015 mags should be added in quadrature

\begin{tabular}{|c|c|c|c|c|c|c|c|c|c|c|c|c|c|c|c|}
\hline \multirow[t]{2}{*}{ Name } & \multicolumn{3}{|c|}{$\bar{N}$} & \multicolumn{3}{|c|}{$8.7 \mu \mathrm{m}$} & \multicolumn{3}{|c|}{$9.8 \mu \mathrm{m}$} & \multicolumn{3}{|c|}{$12.5 \mu \mathrm{m}$} & \multicolumn{3}{|c|}{$Q$} \\
\hline & mag & $\mathrm{sd}$ & no & mag & $\mathrm{sd}$ & no & mag & $\mathrm{sd}$ & no & mag & $\mathrm{sd}$ & no & mag & $\mathrm{sd}$ & no \\
\hline HR 1457 & -3.000 & 0.004 & 10 & -2.949 & 0.003 & 09 & -3.026 & 0.004 & 09 & -3.052 & 0.003 & 09 & -3.071 & 0.004 & 09 \\
\hline HR 2077 & 1.329 & 0.009 & 01 & 1.334 & 0.013 & 01 & 1.323 & 0.015 & 01 & 1.328 & 0.038 & 01 & 1.389 & 0.068 & 01 \\
\hline HR 2335 & 2.296 & 0.008 & 01 & 2.329 & 0.008 & 01 & 2.278 & 0.015 & 01 & & & & 2.289 & 0.049 & 01 \\
\hline HR 2443 & 1.699 & 0.010 & 01 & 1.697 & 0.006 & 01 & 1.707 & 0.010 & 01 & & & & 1.694 & 0.053 & 01 \\
\hline HR 2459 & 1.396 & 0.009 & 01 & 1.434 & 0.008 & 01 & 1.385 & 0.010 & 01 & 1.455 & 0.043 & 01 & 1.344 & 0.044 & 01 \\
\hline HR 2533 & 2.181 & 0.008 & 01 & 2.228 & 0.005 & 01 & 2.149 & 0.010 & 01 & 2.217 & 0.018 & 01 & 2.128 & 0.020 & 01 \\
\hline HR 2970 & 1.528 & 0.008 & 01 & 1.526 & 0.005 & 01 & 1.524 & 0.009 & 01 & & & & 1.541 & 0.043 & 01 \\
\hline HR 3145 & & & & 1.305 & 0.009 & 01 & 1.285 & 0.010 & 01 & & & & 1.265 & 0.039 & 01 \\
\hline HR 3304 & 2.232 & 0.008 & 01 & 2.270 & 0.008 & 01 & 2.214 & 0.011 & 01 & 2.230 & 0.028 & 01 & 2.137 & 0.036 & 01 \\
\hline HR 3738 & 1.931 & 0.006 & 01 & 1.973 & 0.005 & 01 & 1.907 & 0.008 & 01 & & & & 1.893 & 0.024 & 01 \\
\hline HHR 3834 & 1.413 & 0.009 & 02 & 1.427 & 0.010 & 02 & 1.399 & 0.014 & 02 & 1.411 & 0.027 & 02 & 1.371 & 0.052 & 02 \\
\hline HR 3939 & 1.894 & 0.009 & 02 & 1.933 & 0.007 & 02 & 1.873 & 0.009 & 02 & 1.884 & 0.013 & 02 & 1.832 & 0.015 & 01 \\
\hline HHR 4094 & 0.255 & 0.012 & 01 & 0.291 & 0.009 & 01 & 0.222 & 0.007 & 01 & 0.168 & 0.012 & 01 & 0.220 & 0.048 & 01 \\
\hline HHR 4232 & 0.142 & 0.005 & 01 & 0.161 & 0.005 & 01 & 0.139 & 0.007 & 01 & 0.103 & 0.016 & 01 & 0.140 & 0.012 & 01 \\
\hline HR 4335 & 0.296 & 0.007 & 02 & 0.303 & 0.008 & 02 & 0.295 & 0.008 & 02 & 0.306 & 0.018 & 02 & 0.258 & 0.020 & 02 \\
\hline HHR 4402 & 0.837 & 0.013 & 02 & 0.888 & 0.010 & 02 & 0.810 & 0.011 & 02 & 0.791 & 0.026 & 02 & 0.768 & 0.086 & 02 \\
\hline HR 4701 & 1.948 & 0.009 & 01 & 1.982 & 0.011 & 01 & 1.935 & 0.019 & 01 & 1.939 & 0.043 & 01 & 1.899 & 0.089 & 01 \\
\hline HHR 4728 & 2.794 & 0.010 & 01 & 2.794 & 0.007 & 01 & 2.804 & 0.008 & 01 & 2.804 & 0.014 & 01 & 2.864 & 0.037 & 01 \\
\hline HR 4954 & 1.150 & 0.008 & 02 & 1.204 & 0.007 & 02 & 1.135 & 0.008 & 02 & 1.129 & 0.014 & 02 & 1.099 & 0.020 & 02 \\
\hline HR 5315 & 0.908 & 0.008 & 01 & 0.929 & 0.004 & 01 & 0.884 & 0.005 & 01 & 0.883 & 0.012 & 01 & 0.865 & 0.006 & 01 \\
\hline HR 5340 & -3.154 & 0.003 & 12 & -3.133 & 0.002 & 13 & -3.168 & 0.003 & 13 & -3.175 & 0.006 & 13 & -3.182 & 0.003 & 12 \\
\hline HR 5616 & 1.501 & 0.007 & 02 & 1.518 & 0.007 & 02 & 1.488 & 0.011 & 02 & 1.471 & 0.018 & 01 & 1.498 & 0.028 & 02 \\
\hline HR 5622 & 1.338 & 0.008 & 01 & 1.379 & 0.007 & 01 & 1.317 & 0.012 & 01 & 1.271 & 0.025 & 01 & 1.260 & 0.028 & 01 \\
\hline HR 6108 & 1.610 & 0.010 & 02 & 1.642 & 0.004 & 02 & 1.573 & 0.007 & 02 & 1.577 & 0.013 & 02 & 1.551 & 0.019 & 02 \\
\hline HR 6159 & 1.094 & 0.009 & 01 & 1.138 & 0.007 & 02 & 1.070 & 0.007 & 02 & 1.054 & 0.012 & 02 & 1.009 & 0.013 & 02 \\
\hline HR 6705 & -1.426 & 0.008 & 02 & -1.394 & 0.009 & 02 & -1.470 & 0.010 & 02 & -1.472 & 0.023 & 02 & -1.513 & 0.011 & 02 \\
\hline
\end{tabular}

Table 3. The magnitudes in the 5 IRTF filters of the remaining 4 IRTF standard stars not included in the ISO calibration lists

\begin{tabular}{|c|c|c|c|c|c|c|c|c|c|c|c|c|c|c|c|}
\hline \multirow[t]{2}{*}{ Name } & \multicolumn{3}{|c|}{$\bar{N}$} & \multicolumn{3}{|c|}{$8.7 \mu \mathrm{m}$} & \multicolumn{3}{|c|}{$9.8 \mu \mathrm{m}$} & \multicolumn{3}{|c|}{$12.5 \mu \mathrm{m}$} & \multicolumn{3}{|c|}{$Q$} \\
\hline & mag & sd & no & mag & sd & no & mag & $\mathrm{sd}$ & no & mag & $\mathrm{sd}$ & no & mag & $\mathrm{sd}$ & no \\
\hline$\overline{\text { HR } 2061}$ & -5.079 & 0.011 & 03 & -4.794 & 0.011 & 04 & -5.262 & 0.006 & 04 & -5.299 & 0.007 & 03 & -5.700 & 0.010 & 04 \\
\hline HR 2990 & -1.216 & 0.015 & 01 & -1.214 & 0.015 & 01 & -1.217 & 0.015 & 01 & -1.234 & 0.013 & 01 & & & \\
\hline HR 4069 & -0.993 & 0.023 & 01 & -0.925 & 0.005 & 02 & -1.005 & 0.008 & 02 & -1.086 & 0.025 & 02 & -1.071 & 0.030 & 01 \\
\hline HR 4534 & 1.921 & 0.005 & 02 & 1.920 & 0.005 & 02 & 1.917 & 0.006 & 02 & 1.926 & 0.006 & 02 & 1.857 & 0.069 & 02 \\
\hline
\end{tabular}

not include the error in the zero points, which is discussed in more detail in the following section.

For completeness, Table 3 gives the measured magnitudes for the 4 IRTF standards which were are not in the ISO calibration set. Of these stars we note that HR 2061 and HR 4069 are listed in the Bright Star Catalogue as variables. Further HR 2061, although very bright, is a poor standard as it has a significant IR excess so the flux densities are difficult to predict.

\subsection{Probable error in the zero points}

The absence of well calibrated photometry for all of the IRTF filters makes a direct validation of the zero points difficult, so more indirect means have been used.

\subsubsection{The $10 \mu \mathrm{m}$ colours}

In setting the zero points each filter was dealt with individually however, from the definition of the zero points, it is clear that any photospheric A0V star should have colours of 0.00 at all wavelengths. Hence for any of the stars measured, the colours using the IRTF filters should be also very close to zero as all are near the Rayleigh-Jeans part of their spectra. Unfortunately, the $\mathrm{SiO}$ bands means that at $8.7 \mu \mathrm{m}$ and $N$, and to a lesser extent at $9.8 \mu \mathrm{m}$, the cooler stars in the sample will be significantly below the continuum flux density. However, there are sufficient hotter stars to determine the position of the zero colours. In Fig. 4 are plotted the colours using the $9.8 \mu \mathrm{m}$ filter as the common wavelength. Although there is some $\mathrm{SiO}$ present, the accuracy of the photometry is better than in the $12.5 \mu \mathrm{m}$ or $Q$ filters. The error bars are calculated from Table 2. The solid line is the predicted colour from the models (i.e. no $\mathrm{SiO}$ ). The solid square marks the position of HR 1457 and the solid triangle HR 5340, which were the main calibration stars.

If there were a large relative error between the zero point of the $9.8 \mu \mathrm{m}$ and that of another filter the values would be offset away from the predicted line, and in this case away from zero. As can be seen, once the $\mathrm{SiO}$ is allowed for, the bulk of the sources are within $1 \sigma$ of the predicted colour (all are within $2 \sigma$ ) with the average being very close to zero. The presence of the $\mathrm{SiO}$ makes an exact figure difficult to calculate but the figures suggest that the error in the zero points add no more than $1 \%$ to 


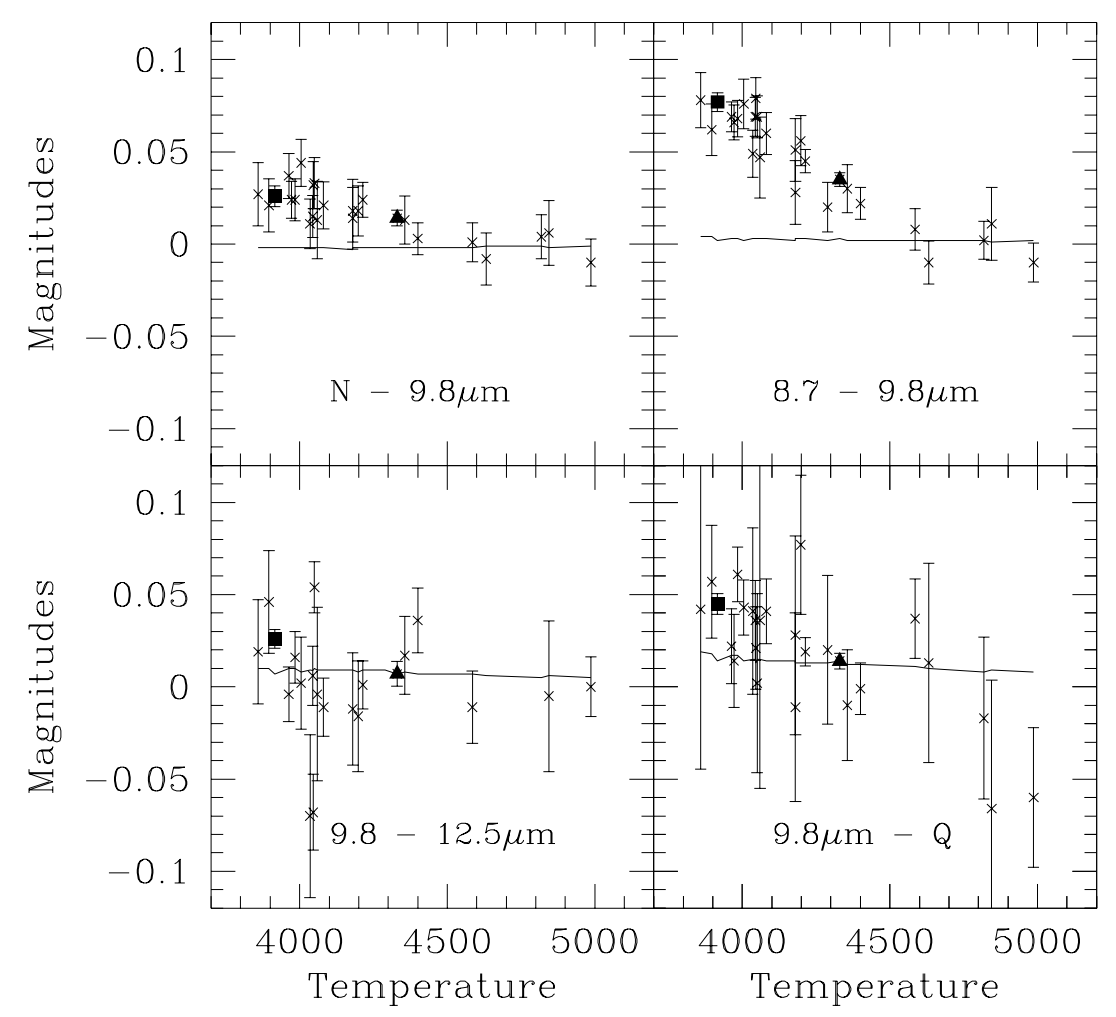

Fig. 4. The measured and predicted colours for the IRTF filters taking the $9.8 \mu \mathrm{m}$ filter as common. The predicted colours is the continuous line. The main calibration stars are marked as a solid square (HR 1457) and a solid triangle (HR 5340)

Table 4. The measured and predicted colours for the IRTF filters taking the $9.8 \mu \mathrm{m}$ filter as common. The predicted colours is the continuous line. The main calibration stars are marked as a solid square (HR 1457) and a solid triangle (HR 5340)

\begin{tabular}{lcccccc}
\hline & & $N$ & & $c$ & \\
& This work & Tokunaga & Rieke & This work & Tokunaga & Rieke \\
\hline HR 1457 & -3.000 & $-3.03 \pm 0.014$ & $-3.03 \pm 0.02$ & -3.071 & $-3.09 \pm 0.02$ & $-3.09 \pm 0.02$ \\
HR 2990 & -1.216 & & $-1.23 \pm 0.04$ & & & \\
HR 5340 & -3.154 & $-3.17 \pm 0.014$ & $-3.17 \pm 0.02$ & -3.182 & $-3.13 \pm 0.03$ & $-3.21 \pm 0.02$ \\
HR 6705 & -1.426 & & $-1.50 \pm 0.04$ & -1.513 & & $-1.56 \pm 0.04$ \\
\hline
\end{tabular}

the error of the colours. Clearly this is just a relative error, but indicates that the zero points are internally consistent.

\subsubsection{Comparison with other 10 micron data}

Tokunaga (1984) has published magnitudes for a number of stars in the IRTF $N$ and $Q$ filters. These are calibrated against Vega, which is defined as having a magnitude of 0.00. A second source is Rieke et al. (1985). Rieke et al. provide a comparison between their system and that of Tokunaga and conclude at $10 \mu \mathrm{m}$ the maximum difference for the stars in common is 0.02 magnitudes. However, Rieke et al. have defined their zero point to agree with that Tokunaga value.

Table 4 lists the magnitudes for the brightest, multiply measured stars in this work which are also in Tokunaga $\&$ Rieke et al. There are two other stars in common how- ever HR 4069 is listed as being variable and HR 4534 is amongst the faintest presented here (even if these stars were included the comparison presented below would be very similar). The comparison magnitudes have the errors which include the errors in the zero points. Using weighted means the difference between the zero points are

$$
\begin{aligned}
& N-N_{\text {Tokunaga }}=0.023 \pm 0.01 \\
& Q-Q_{\text {Tokunaga }}=-0.015 \pm 0.02 \\
& N-N_{\text {Rieke }}=0.028 \pm 0.014 \\
& Q-Q_{\text {Rieke }}=0.024 \pm 0.014
\end{aligned}
$$

Note the $Q_{\text {Tokunaga }}$ has a positive $N-Q$ colour for HR 5340 , which is unreasonable for a $\mathrm{K}$ giant. If the $Q_{\text {Tokunaga }}$ 
for HR 5340 is ignored then the $Q-Q_{\text {Tokunaga }}=0.02$, however, this is based solely on HR 1457.

Hence there appears to be a small offset between the $N$ and $Q$ zero points used here and those of Tokunaga/Rieke, however it is only, at most, at the $2 \sigma$ level. From the discussion on the colours we suggest that the simplest method to convert all of the magnitudes presented here to be compatible with the zero points as defined by Rieke et al. or Tokunaga is to subtract 0.024 from all the magnitudes given in Tables 2 and 3 .

\subsubsection{Comparison with spectral composite/templates}

In Cohen et al. (1995a) a method is presented to construct complete 1.2 to $35 \mu \mathrm{m}$ spectra of $\mathrm{K}$ giants by splicing together measured spectral fragments. The spectrum that is produced is linked though Sirius to the absolute Vega model used in this work (Cohen et al. 1992). Therefore, apart from the last step of conversion to flux density, these spectral composites are independent of the Kurucz model grid and will include the $\mathrm{SiO}$ features. As the composites are complete spectra it is possible to generate magnitudes for any filter set in the wavelength range and so give, in effect, measured magnitudes.

The difference between the magnitudes presented here and those from the spectral composites (e.g. $N_{\mathrm{c}}$ ) for the main calibration stars (i.e. HR 1457, HR 5340 and HR 6705) are.

$N-N_{\mathrm{c}}=-0.010 \pm 0.010$

$[8.7]-[8.7]_{\mathrm{c}}=+0.005 \pm 0.008$

$[9.8]-[9.8]_{\mathrm{c}}=+0.014 \pm 0.008$

$[12.5]-[12.5]_{\mathrm{c}}=-0.013 \pm 0.010$

$Q-Q_{\mathrm{c}}=-0.003 \pm 0.007$.

In Cohen et al. (1996) a method of extending the spectral composites to fainter stars is developed. These are called spectral templates and assume that the spectral shape is defined from the spectral type and luminosity class and the flux density level set using near IR photometry. These are not as accurate as the composites but there are many more stars available for comparison. As templates are not as accurate as the composites and the stars are not as well measured the template values given below should not be used to convert the zero points to the Cohen system. Rather they give an indication of the internal consistency.

The differences between the magnitudes presented here and the magnitudes predicted from spectral templates (e.g. $N_{\mathrm{T}}$ ) for 9 stars in the list for which templates exist are:

$N-N_{\mathrm{T}}=-0.017 \pm 0.015$

$$
\begin{aligned}
& {[8.7]-[8.7]_{\mathrm{T}}=-0.001 \pm 0.011} \\
& {[9.8]-[9.8]_{\mathrm{T}}=+0.019 \pm 0.013} \\
& {[12.5]-[12.5]_{\mathrm{T}}=+0.02 \pm 0.020} \\
& Q-Q_{\mathrm{T}}=+0.004 \pm 0.015
\end{aligned}
$$

\subsubsection{Assumed error in the zero points}

From the above it would appear that the zero points are close to those defined at the outset, i.e. Vega having 0.00 mags in all filters. The maximum error when comparing with other determinations of the zero points is $0.028 \mathrm{mag}$, with the average error being less than this. It is very difficult to accurately determine the true error in the zero points as each of the above data sets used for reference has possible systematic errors which are difficult to evaluate. Taking the average difference as being the error in the zero points, gives a probable error in the zero points for the data set presented here of about 0.015 magnitudes (rms) in all filters.

\subsection{Conversion to flux density}

As the data presented here are based on broad band filter photometry, the flux density given are the isophotal flux $F_{\text {iso }}$.

$$
\begin{aligned}
F_{\text {iso }} & =\frac{\int_{0}^{\infty} S_{\lambda} f_{\lambda} \mathrm{d} \lambda}{\int_{0}^{\infty} S_{\lambda} \mathrm{d} \lambda} \\
\text { i.e. } & \\
F_{\text {iso }} & =\frac{\text { In Band Flux }}{\int_{0}^{\infty} S_{\lambda} \mathrm{d} \lambda} .
\end{aligned}
$$

Table 5. The isophotal wavelengths and fluxes for Vega in the 5 IRTF filters

\begin{tabular}{ccccc}
\hline Filter & $\lambda_{\text {iso }}$ & $\mathrm{Wm}^{-2} \mu \mathrm{m}^{-1}$ & $\nu_{\text {iso }}$ & $\mathrm{Jy}$ \\
\hline$N$ & $10.471 \mu \mathrm{m}$ & $9.632 E-13$ & $2.919 E 13$ & 36.55 \\
$8.7 \mu \mathrm{m}$ & $8.789 \mu \mathrm{m}$ & $1.924 E-12$ & $3.415 E 13$ & 49.59 \\
$9.8 \mu \mathrm{m}$ & $9.863 \mu \mathrm{m}$ & $1.221 E-12$ & $3.045 E 13$ & 39.64 \\
$12.5 \mu \mathrm{m}$ & $12.454 \mu \mathrm{m}$ & $4.841 E-13$ & $2.409 E 13$ & 25.07 \\
$Q$ & $20.130 \mu \mathrm{m}$ & $7.182 E-14$ & $1.511 E 13$ & 9.947 \\
\hline
\end{tabular}

In Table 5 is presented the isophotal fluxes for Vega in the 5 IRTF filters. The fluxes are also given in terms of Jy which are calculated using the above equation but in frequency space. The wavelength at which the continuum spectrum of a star has $F_{\text {iso }}$ is known as the isophotal wavelength, $\left(\lambda_{\text {iso }}\right)$. Table 5 lists the $\lambda_{\text {iso }}$ for Vega in the 5 filters. It also lists the $\nu_{\text {iso }}$ which is the frequency at which the continuum spectrum has the stated flux density in Jy. It should be noted that $\lambda_{\text {iso }}$ is not the same as $\nu_{\text {iso }}$. For 
a full discussion of the significance of isophotal fluxes and wavelengths, refer to Golay (1974).

The isophotal flux for any star, $s$, is then

$F_{\text {iso }}, s=F_{\text {iso,vega }} 10^{-0.4 m_{s}}$

where $m_{s}$ is the apparent magnitude of the star.

In Table 6 are presented the isophotal fluxes in $\mathrm{Wm}^{-2} \mu \mathrm{m}^{-1}$ and Jy for the 26 stars. The error in the flux is also given, which is determined from the error in the measurement and the zero point. As stated previously, the error in the absolute Vega flux has been ignored.

\section{Discussion}

\subsection{Validity of using models to extrapolate mid IR magnitudes from near IR data}

The stars were chosen as potential infrared standards, so they are predictable (i.e. non-variable and single) and are well measured at other wavelengths. Hence the results presented here provide a good test on the validity of using models to extrapolate mid infrared magnitudes from near infrared data.

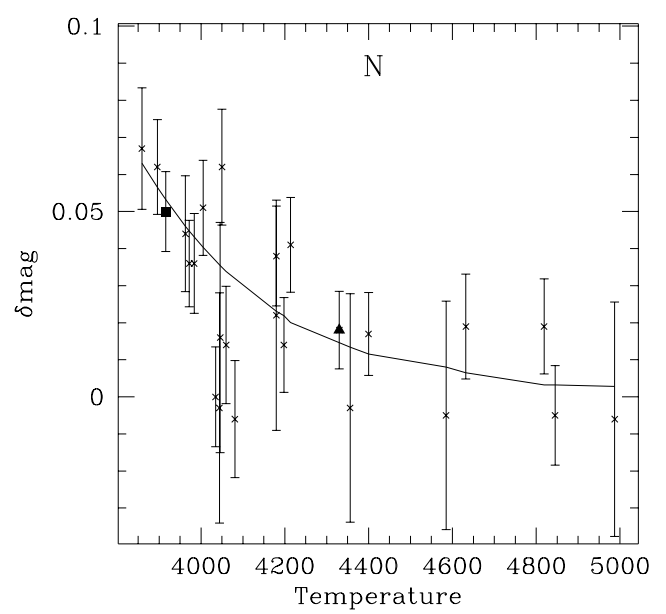

Fig. 5. The difference between the predicted and measured $N$ magnitude

In Figs. 5-9 are presented the difference between the magnitudes predicted using the model SEDs and the measured magnitude. The main calibrators are marked as a solid square (HR 1457) and a solid triangle (HR 5340). The error bars include the errors from the measurement and the predicted magnitude. The latter comes primarily from the error in the near IR magnitudes used to normalise the model SED, typically this is of order $1 \%$ but a few of the stars are less well measured and for these the error could reach 2 to $3 \%$. The $\mathrm{SiO}$ bands are not included in the model grid used, so in order to allow for this a low order polynomial fit has been put through the difference

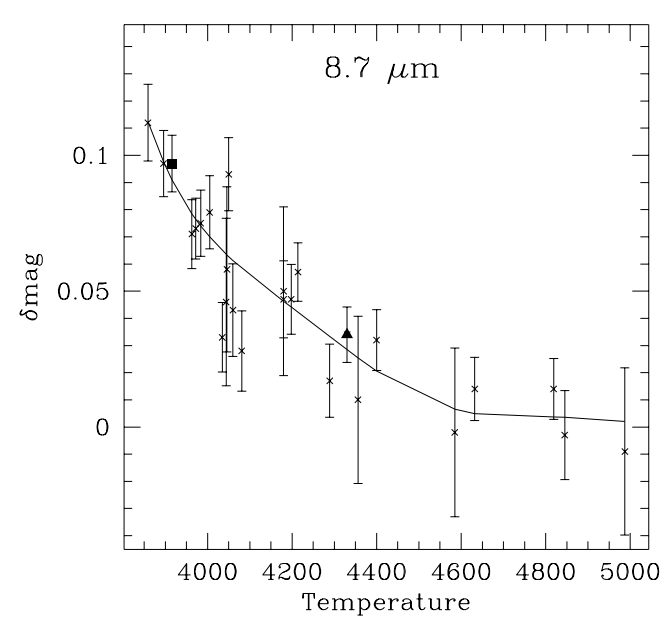

Fig. 6. The difference between the predicted and measured $8.7 \mu \mathrm{m}$ magnitude

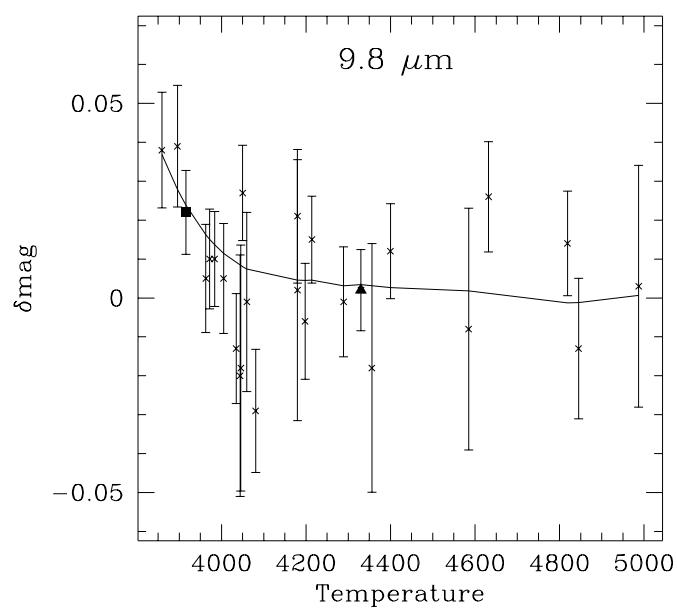

Fig. 7. The difference between the predicted and measured $9.8 \mu \mathrm{m}$ magnitude

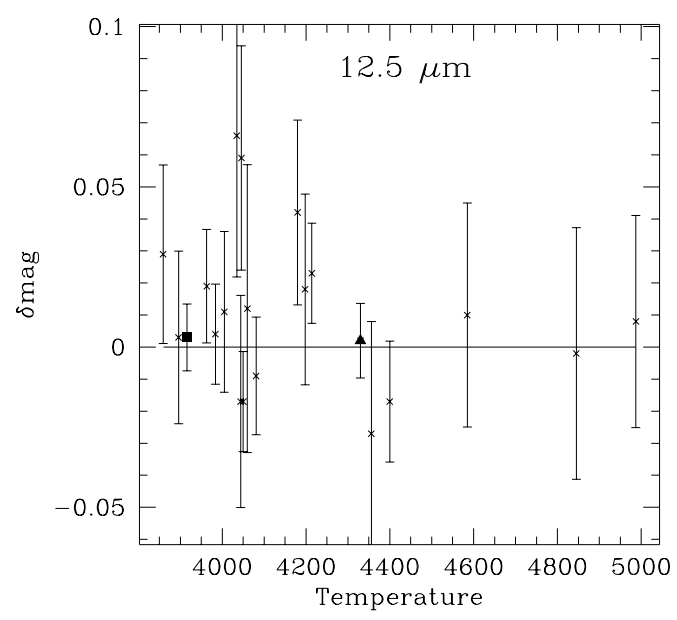

Fig. 8. The difference between the predicted and measured $12.5 \mu \mathrm{m}$ magnitude 
Table 6. The fluxes $\mathrm{Wm}^{-2} \mu \mathrm{m}^{-1}$ and Jy for the 5 IRTF filters. The percentage error is also given and includes the error in the zero points

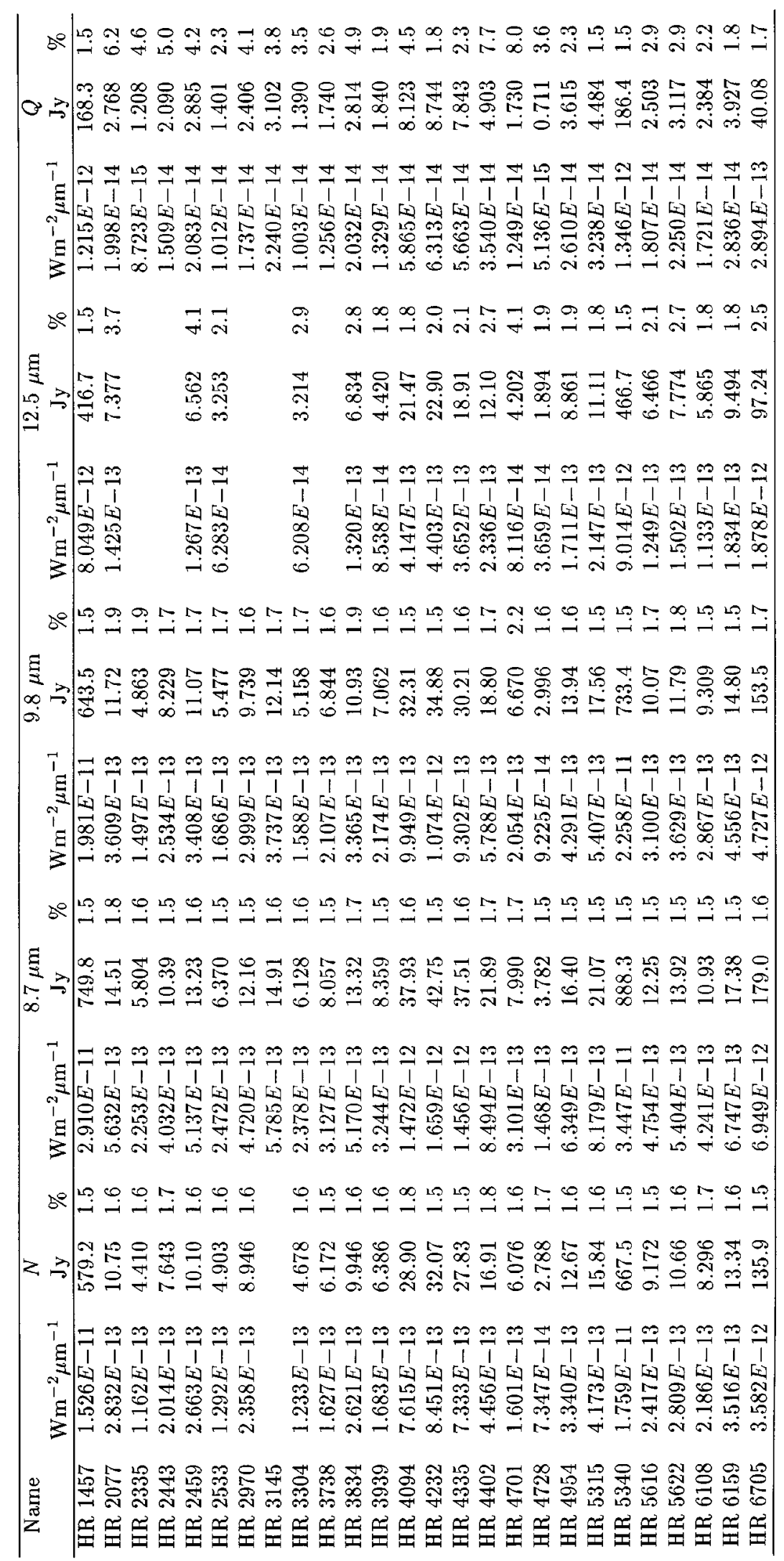




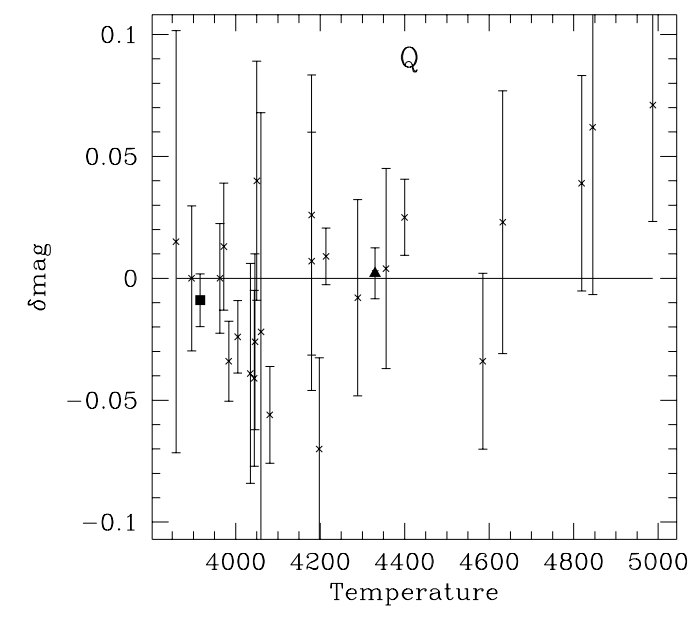

Fig. 9. The difference between the predicted and measured $Q$ magnitude

for the $N, 8.7$ and $9.8 \mu \mathrm{m}$ filters. The $\mathrm{SiO}$ is not significant in the $12.5 \mu \mathrm{m}$ and $Q$ filters so the line was drawn through zero difference.

It can be seen in Figs. 5-9 that the fitted line goes through the majority of the error bars. The fitted line was subtracted from the data points and the standard deviation of the difference calculated (Table 7). In calculating the standard deviation the three points with the largest errors in the predicted magnitudes were ignored because the error comes from the near IR magnitude used to normalise the SED. It is intended to improve the near IR measurements of these stars, but this has not yet been possible.

Table 7. The standard deviation between the predicted and measured magnitudes for each filter after the $\mathrm{SiO}$ has been allowed for

\begin{tabular}{ccc}
\hline Filter & sd & comment \\
\hline$N$ & 0.014 & \\
$8.7 \mu \mathrm{m}$ & 0.013 & \\
$9.8 \mu \mathrm{m}$ & 0.014 & \\
$12.5 \mu \mathrm{m}$ & 0.023 & $(0.017$ if worst 2 points are deleted $)$ \\
$Q$ & 0.035 & \\
\hline
\end{tabular}

The size of the standard deviation of the difference between the measured magnitude and the predicted magnitude indicates that the errors in the measurements presented in Table 2 are reasonable and that the random errors in the SEDs are of the order quoted. The previous discussion on the zero points indicates that there is a possible error in the zero points of about 0.015 mags although this is at the same level as the errors used to compare with other zero points so it is difficult to assign a significance to it. We therefore conclude that the method of determining the zero points for the IRTF 10 and $20 \mu \mathrm{m}$ filters presented here is valid within the quoted errors. Further, the method should be valid for any filter within the wavelength range covered here, although if measurements are to be taken in the $\mathrm{SiO}$ bands then stars hotter than $4500 \mathrm{~K}$ should be used. The target accuracy at $10 \mu \mathrm{m}$ for the ISO calibration programme was $5 \%$. This data set shows that the difference between the measured and predicted flux densities, including the error in the zero point, is about $2 \%$ over the wavelength range covered here. In fact, at $Q$ the predicted magnitudes are in general more accurate than the measured ones.

\subsection{The SiO bands}

Although the $\mathrm{SiO}$ bands at 8 to $10 \mu \mathrm{m}$ are not included in the stellar models, the data presented here clearly show that the $\mathrm{SiO}$ does have a significant effect for stars cooler than $4500 \mathrm{~K}$. For the coolest stars in this sample the error between the measured and predicted magnitudes is as high as 0.11 magnitudes in the $8.7 \mu \mathrm{m}$ filter.

Figures 5-7 indicate that for stars with effective temperatures between $3850 \mathrm{~K}$ and $4500 \mathrm{~K}$ the effect of the $\mathrm{SiO}$ can be approximated to a linear regression in the $\mathrm{N}, 8.7$ and $9.8 \mu \mathrm{m}$ filters. The difference between the measured and predicted magnitudes are:

$$
\begin{aligned}
& N_{\text {measured }}-N_{\text {predicted }}=9.010^{-5}\left(4500-T_{\mathrm{e}}\right) \\
& {[8.7]_{\text {measured }}-[8.7]_{\text {predicted }}=1.710^{-4}\left(4500-T_{\mathrm{e}}\right)} \\
& {[9.8]_{\text {measured }}-[9.8]_{\text {predicted }}=4.010^{-5}\left(4500-T_{\mathrm{e}}\right) .}
\end{aligned}
$$

With the advent of infrared observatories such as ISO and the availability of powerful mid IR instrumentation on the latest generation of 8 and $10 \mathrm{~m}$ class telescopes, the mid IR will gain in importance. If stellar models are to accurately represent the flux density between 8 and $10 \mu \mathrm{m}$ for stars cooler than $4500 \mathrm{~K}$ it is imperative that the $\mathrm{SiO}$ bands be included.

\subsection{Stellar photometry in the narrow band $10 \mu \mathrm{m}$ filters}

As can be seen from Tables 2 and 6, the errors in the 8.7, 9.8 and $12.5 \mu \mathrm{m}$ filters are comparable to those in the full $N$ filter and so in terms of accuracy there is nothing lost by using the narrow bands. In fact, as the narrow band filters use a smaller portion of the atmospheric window, they are much less susceptible to changes in atmospheric condition than the broad band $N$. This should be particularly true for the $8.7 \mu \mathrm{m}$ filter which appears to be relatively clean (see Fig. 1).

As the $N$ filter covers from 8.2 to $13.5 \mu \mathrm{m}$, about half of the wavelength range is affected by the $\mathrm{SiO}$. Therefore, the magnitudes have to be corrected for studies which require a continuum flux, e.g. the IRFM. Clearly these studies would be better using the $12.5 \mu \mathrm{m}$ filter, at least for 
the cool stars, as it is almost purely continuum. However, when the object of the observations is to measure $\mathrm{SiO}$ then the effect on the $8.7 \mu \mathrm{m}$ filter magnitude is about twice that on the $N$ magnitude (see above). Further, the difference in magnitude between the 8.7 and $12.5 \mu \mathrm{m}$ filters would directly give the amount of $\mathrm{SiO}$ in the star.

There are significant advantages in interpretation of the narrow band data. The $N$ filter has a very low spectral resolution and so the $\lambda_{\text {iso }}$ changes significantly with spectral type; the $\lambda_{\text {iso }}$ for a K5III is about $1 \%$ longer than an A0V. However, for the narrow band filters the change in $\lambda_{\text {iso }}$ is at most $0.2 \%$ between A0 and K5. The effective wavelength is an approximation to $\lambda_{\text {iso }}$ (see Golay 1974) but often the effective wavelength is quoted in preference as it has a simpler definition. For the narrow band filters the effective wavelength is within $0.2 \%$ of the $\lambda_{\text {iso }}$ and hence does provide a good approximation. However, for the broad band $N$ the difference is about $3 \%$. As the flux changes as $\lambda^{-4}$, a $3 \%$ change in wavelength is equivalent to a $12 \%$ change in flux. Hence, if the $N$ isophotal flux were given at the $N$ effective wavelength there would be, in effect, an error of $12 \%$ in the flux.

\section{Conclusions}

Presented is $N, 8.7 \mu \mathrm{m}, 9.8 \mu \mathrm{m} 12.5 \mu \mathrm{m}$ and $Q$ photometry on a set of 26 stars which were measured on the IRTF. The errors in the measurements are of the order 1 to $1.5 \%$ in the $N, 8.7 \mu \mathrm{m}, 9.8 \mu \mathrm{m} 12.5 \mu \mathrm{m}$ and $3 \%$ at $Q$. The zero points in the 5 filters have been set using near IR photometry and the Kurucz model grid to predict the IR magnitudes in the 5 filters. The probable error in the derived zero points is 0.015 mags which is comparable to the current error in the more direct methods of determining the zero points in this wavelength range. The main problem with the method is that the Kurucz model grid, which was used here, does not include the $\mathrm{SiO}$ bands and so stars hotter than $4500 \mathrm{~K}$ are needed to set the zero points in the $N, 8.7 \mu \mathrm{m}$ and $9.8 \mu \mathrm{m}$ filters. The results presented here do validate part of the proposed method for calibration of ISO, at least between 8 and $26 \mu \mathrm{m}$. These 26 stars now qualify as reliable, self-consistent set of mid IR standards.
Acknowledgements. We would like to thank the staff of the IRTF for their assistance during the observations. We thank Dr. M. Cohen for providing access to his composite spectra and spectral templates well as helpful comments on an original draft of the paper, and also thank the referee, A. Tokunaga, for useful suggestions.

\section{References}

Aumann, et al., 1985, ApJ 278, L23

Blackwell D.E., Petford A.D., Arribas S., Haddock D.J., Selby M.J., 1990, A\&A 232, 396

Blackwell D.E., Lynas-Gray A.E., Petford A.D., 1991, A\&A 245,567

Cayrel de Strobel G., Hauck B., Francois P., et al., 1992, A\&AS 95, 273

Cohen M., Walker R.G., Barlow M.J., Deacon J.R., 1992, AJ 104,1650

Cohen M, Witteborn F.C., Walker R.G., Bregman J.D., Wooden D.H., 1995a, AJ 110, 275

Cohen M, 1995b, (private communication)

Golay M., 1974, Introduction to Astronomical Photometry, Vol. 41 in the Astrophysics and Space Science Library. Reidel, Dordrecht, pp. 39-46

Hammersley P.L., et al., 1997, (in preparation)

Hanner M.S., Tokunaga A.T., 1991, Comets in the Post-Halley Era, Vol. 1 Newburn R.L., et al., (eds.). Kluwer, Holland, p. 67

Jourdain de Muizon, M., Habing, H.J., 1991, in Infrared Astronomy with ISO, Encrenaz Th. and Kessler M.F. (eds.). Nova Science Publishers, p. 129

Kurucz R.L., 1991, Precision Photometry: Astrophysics of the Galaxy, Davis Philip A.G., Upgren A.R. and Janes K.A., (eds.). L. Davis Press, Schenectady, p. 27

Kurucz R.L., 1993, data on magnetic tape

Mégessier C., 1994, A\&A 289, 202

Mégessier C., 1995, A\&A 296, 771

Rieke G.H., Lebofsky M.J., Low F.J., 1985, AJ 90, 900

Selby M.J., Hepburn I., Blackwell D.E., et al., 1988, A\&AS 74, 127

Tokunaga A.T., 1984, AJ 89, 172

Turon C., et al., 1992, Hipparcos Input Catalogue, ESA, SP1136, 7 volumes

van der Bliek N.S., et al., 1992, ESO Messenger 70, 28

van der Bliek N.S., Manfroid J., Bouchet P., 1996, A\&AS 119, 547 